\title{
Extraperitoneal Leakage as a Possible Explanation for Failure of One-Time Intraperitoneal Treatment in Ovarian Cancer
}

\author{
Angèle L.M. Oei, ${ }^{1}$ Leon F.A.G. Massuger, ${ }^{1}$ and Wim J.G. Oyen ${ }^{2}$ \\ From the Departments of ${ }^{1}$ Obstetrics and Gynecology and ${ }^{2}$ Nuclear Medicine, Radboud University \\ Nijmegen Medical Centre, Nijmegen, The Netherlands
}

\begin{abstract}
We conducted a single-arm study to determine the biodistribution of intraperitoneally (i.p.) administered ${ }^{90}$ yttrium-labeled murine monoclonal antibody HMFG1 $\left({ }^{90} Y\right.$-muHMFG1) in patients with advanced stage ovarian cancer. Seventeen (17) patients in complete clinical remission for epithelial ovarian cancer were included. After completion of chemotherapy, a mixture of ${ }^{111}$ indium-labeled muHMFG1 (imaging) and ${ }^{90} Y$-muHMFG1 (therapy) was i.p. administered by a surgically placed, indwelling i.p. catheter. Planar and single-photon emission computed tomography images were recorded to determine the distribution of the study medication during the first 6 days postinjection. Of the first 3 patients, 2 patients had extraperitoneal leakage of up to 50\% of the injected dose within 24 hours after injection of the study medication. Extraperitoneal leakage was mainly seen in the retroperitoneal spaces covering the upper and lower quadrant of the abdomen. After adjustments in the procedure, leakage was observed in 2 of the remaining 14 patients. Extraperitoneal leakage of i.p. administered therapy does occur. Such leakage would reduce the locally delivered dose of a drug and could potentially have a negative impact on therapeutic efficacy. Given the potential attraction of developing i.p. treatments for intra-abdominal cancer, the observations in this study need to be taken into consideration.
\end{abstract}

Key words: intraperitoneal, monoclonal antibody, ovarian cancer, HMFG1

\section{INTRODUCTION}

Despite advances in the treatment of ovarian cancer, it is still the most deadly of the gynecologic malignancies. In the majority of cases, it is diagnosed at an advanced stage, when 5-year survival is $25 \%-30 \%{ }^{1}$ Although there is a high response

\footnotetext{
Address reprint requests to: Angèle L.M. Oei; Department of Obstetrics and Gynecology, Radboud University Nijmegen Medical Centre; P.O. Box 9101, 6500 HB Nijmegen, The Netherlands; Tel.: +31-62-4148448; Fax: +31-24-3668597

E-mail: A.Oei@obgyn.umcn.nl
}

rate to cytoreductive surgery followed by platinum-based chemotherapy, the majority of patients develop recurrent disease, particularly in the abdominal cavity. Failure to control intra-abdominal disease remains the primary reason for poor overall outcome.

Intraperitoneal (i.p.) delivery of antineoplastic therapy was first explored in the 1950s, and over the last five decades several forms of intraperitoneal therapy (e.g., i.p. chemotherapy, i.p. radioimmunotherapy, i.p. radioactive P-32 phosphate, and i.p. unlabeled monoclonal antibody $[\mathrm{mAb}]$ therapy) have been investigated in patients with ovarian, colorectal, and gastric cancer. ${ }^{2}$ Ad- 
vantages of i.p. therapy are the achievement of higher local drug concentrations, which can greatly exceed drug concentrations in the plasma, reduced systemic toxicity, prolonged tumor exposure with an assumed greater effect on tumor reduction, and a slow systemic uptake from the peritoneal cavity. ${ }^{3,4}$ The different i.p. therapy forms have shown variable results.

Despite advantages of an i.p. approach, i.p. studies have not yet led to a standardization of i.p. therapy in ovarian cancer. At least three randomized studies have shown a survival benefit for the use of i.p. chemotherapy in ovarian cancer patients, with residual disease smaller than 2 $\mathrm{cm}$ in diameter after debulking surgery. ${ }^{5-7}$ However, toxicity and difficulties inherent to this approach have, until recently, precluded its acceptance into clinical practice. ${ }^{4,8,9}$

In a phase III, randomized, clinical trial, a novel approach using an i.p. injection of the HMFG1 murine mAb radiolabeled with 90yttrium was studied. ${ }^{10}$ HMFG1 is directed toward the MUC1 gene, which is a large, heavily glycosylated mucin expressed on the apical surface of the majority of secretory epithelial cells. ${ }^{11}$ In $90 \%$ of the epithelial ovarian cancers, MUC1 is overexpressed on the cell surface and it is antigenically distinct from normal tissue mucin as a result of underglycosylation or aberrant glycosylation of this protein in cancerous tissue. ${ }^{12,13}$

Parallel to the phase III study, ${ }^{10}$ we conducted an open, single-arm study to determine the biodistribution of the i.p. administered therapeutic agent ${ }^{90}$ yttrium-labeled murine HMFG1 $\left({ }^{90} \mathrm{Y}-\right.$ muHMFG1).

\section{PATIENTS AND METHODS}

Between July 2001 and July 2004, patients in complete clinical remission following cytoreductive surgery and platinum-based chemotherapy for a prior histologic proven diagnosis of epithelial ovarian cancer FIGO stages Ic-IV were enrolled in the Monoclonal Antibody Imaging and Dosimetry Assessment Study (MIDAS). The study was approved by the Institutional Review Board of the Radboud University Nijmegen Medical Centre (Nijmegen, The Netherlands). Written, informed consent was obtained from all patients prior to study entry. Eligibility criteria consisted of proven epithelial ovarian cancer, including primary peritoneal serous carcinoma with ovarian involvement, entry within 4-8 weeks from final administration of chemotherapy, a life expectancy of at least 3 months, an age over 18 years, and adequate bone marrow (leukocyte count $\geq 3 \times 10^{9} / \mathrm{L}, \quad$ platelet count $\geq 100 \times$ $10^{9} / \mathrm{L}$, hemoglobin $10 \mathrm{~g} / \mathrm{dL}$ ), renal (serum creatinine $\leq 177 \mu \mathrm{mol} / \mathrm{L}$ or $20 \mathrm{mg} / \mathrm{L}$ ), and hepatic function (alanine aminotransferase and aspartate aminotransferase not higher than twice the upper limit of normal). Patients were excluded from inclusion in the study if they had known metastases at the time of second-look laparoscopy (SLL) or had prior or concomitant malignancy other than basal cell carcinoma of the skin. Further exclusion criteria were: serious concomitant physical or psychiatric disease, previous exposure to murine antibodies or the presence of human-antimouse antibodies (HAMA) as detected by the central laboratory, and participation in any other experimental treatment program.

During SLL, the trocar for the laparoscope was placed inferior and the trocar for the laparoscopic probe inferior and lateral to the umbilicus. If there was any visible evidence of malignant disease at SLL, the patient was excluded from the study. At negative SLL, the patient was further enrolled in the study and the peritoneal dialysis catheter was placed at the same place as the trocar for the laparoscopic probe and was fixated to the abdominal wall. Administration of the study medication was performed 1 day after SLL through the peritoneal dialysis catheter. Before administering radiolabeled antibody, ${ }^{99} \mathrm{~m}$ technetium $\left({ }^{99 \mathrm{~m}} \mathrm{Tc}\right)$ colloid was injected i.p. to verify an adequate fluid distribution within the peritoneal cavity. Significant loculation of the i.p. cavity - that might preclude adequate distribution of the study medication-was excluded by the initial i.p. administration of $99 \mathrm{~m} \mathrm{Tc}$-colloid. After the administration of ${ }^{99 \mathrm{~m}} \mathrm{Tc}$-colloid-colloid, the patient was moved frequently to ensure adequate intra-abdominal distribution. Subsequently, anterior and posterior planar images were recorded to check the adequate distribution of the radiopharmaceutical to all four abdominal quadrants. When distribution was seen in at least 3 of the 4 abdominal quadrants, $0.5 \mathrm{~L}$ of saline solution, heated to body temperature, was infused. Thereafter, a single therapeutic dose of ${ }^{90} \mathrm{Y}$-muHMFG1 (Antisoma, London, UK; ${ }^{90} \mathrm{Y}$ activity dose 666 $\mathrm{MBq} / \mathrm{m}^{2}$ of body-surface area with a maximum dose, but not exceeding $1110 \mathrm{MBq}$ ) spiked with an imaging dose of $185 \mathrm{MBq}$ of ${ }^{111}$ indiummuHMFG1was injected through the "Y" connector or two-way tap in 30-60 seconds. Al- 
though obviously not necessary for imaging and dosimetry studies, it was decided to offer the therapeutic ${ }^{90} \mathrm{Y}$-muHMFG1 to patients participating in this study, as patients were subjected to all the same invasive procedures as the patients in the active treatment arm of the phase III trial that was recruiting in parallel. During and after the infusion of the radiopharmaceuticals, the saline solution continued to flow until the i.p. space was filled with $1.5 \mathrm{~L}$ of saline solution. After the administration of the study medication, the catheter was removed and the skin was closed. To enhance the distribution of the study medication in the peritoneal cavity, the patient was asked to move gently during the first hour after administration.

Planar and single-photon emission computed tomography (SPECT) images were recorded to determine the distribution of the study medication. On the anterior and posterior images, regions of interest (ROIs) were drawn around the extraperitoneal leakage (corrected for background by an ROI drawn in the adjacent soft tissues in which no leakage was observed) and over the whole body. When drawing the ROIs over the extraperitonel leakage, the inclusion of intra-abdominal activity was carefully avoided. At all time points, the measured counts in ROIs were corrected for the physical decay of ${ }^{111}$ indium. SPECT was performed twice at 48 and 168 hours postinjection for dosimetric calculations (data not shown), but not used for the assessment of leakage. The percentage of leakage was calculated by using the geometric means of the decay-corrected counts in the following formula:

$$
\begin{aligned}
& \sqrt{ }\left(\left(\mathrm{ROI}_{\text {leakage, ant }}-\mathrm{ROI}_{\mathrm{bkgr}, \text { ant }}\right)\right. \\
& \left.\times\left(\mathrm{ROI}_{\text {leakage, post }}-\mathrm{ROI}_{\text {bkgr, post }}\right)\right) \\
& \sqrt{\left(\mathrm{ROI}_{\mathrm{WB}} \text {, ant, } \mathrm{t}=0\right.} \times \mathrm{ROI}_{\mathrm{WB}, \text { post }, \mathrm{t}=0)}
\end{aligned}
$$

Blood sampling and imaging was performed at $1,6,24,48,72,96,120$, and 168 hours after injection of the study medication, with a range of \pm 4 hours. Blood samples were collected, weighed, and counted in a $\gamma$-counter. By simultaneous measurement of a known aliquot of the injected dose, the percentage injected dose per gram $(\% \mathrm{ID} / \mathrm{g})$ was determined for each sample.

For safety purposes, patients had a weekly follow-up for 6 weeks, then at weeks 8 and 12, and 3 months thereafter, up to 1 year posttreatment. The follow-up consisted of a physical examination, as well as blood and urine sampling to determine total activity that was excreted.

\section{Statistical Analyses}

Data analyses were performed by using the SPSS 12.0.1 software packet (SPSS Inc.; Chicago, IL). Differences between characteristics were tested as two-sided with the independent $t$ test. $P$-values $\leq 0.05$ were considered significant.

\section{RESULTS}

A total of 17 patients received the i.p. study medication of the MIDAS study (Table 1). In the first 3 patients, 2 cases of substantial leakage from the peritoneal cavity to extraperitoneal spaces were observed. Patient 1 showed a moderate extraperitoneal leakage of approximately $11 \%$ of the injected study medication into the right-lower quadrant of the abdomen. Patient 3 had a major extraperitoneal leakage of $50 \%$ of the injected activity into the right-upper and -lower quadrant of the abdomen (Fig. 1). These observations resulted in an adjustment of the protocol. After the first 3

\begin{tabular}{|c|c|c|c|c|c|}
\hline FIGO stage & No. of patients & Serous & Papillary & Endometroid & Other \\
\hline $\mathrm{IIb}$ & 1 & 1 & - & - & - \\
\hline IIIa & 2 & 2 & - & - & - \\
\hline IIIb & 6 & 3 & - & 1 & $2^{\mathrm{a}}$ \\
\hline IIIc & 7 & 4 & 2 & 1 & - \\
\hline IV & 1 & 1 & - & - & - \\
\hline
\end{tabular}
patients, the protocol was revised and adjusted with the following measures: first, the trocar in- 


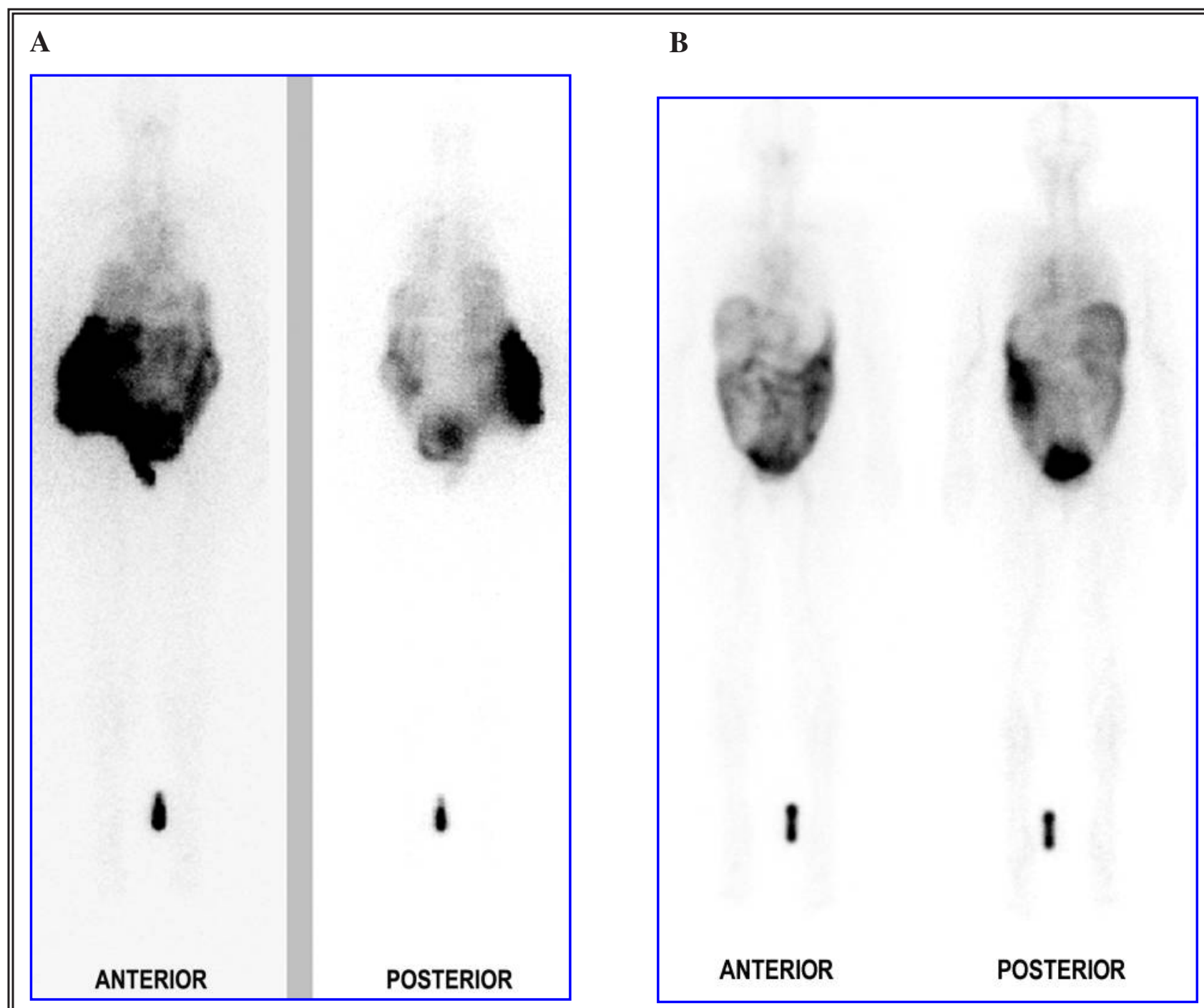

Figure 1. (A) Whole-body images of patient 3 at 24 hours postinjection of ${ }^{111}$ indium-muHMFG1: Major extraperitoneal leakage (50\% of the injected activity leaked into the right-upper and -lower quadrant of the abdomen), and (B) whole-body images of patient 9 at 24 hours postinjection of ${ }^{111}$ indium-muHMFG1 without extraperitoneal leakage.

cision for the laparoscopic probe and catheter was displaced from the lateral to the medial side of the abdomen. Second, the peritoneal dialysis catheter was replaced by a Foley catheter (Euromedical; Lynge, Denmark) to ensure that the abdominal opening would be closed firmly; the balloon of the Foley catheter was inflated, pulled back, and secured against the abdominal wall with sutures before the administration of the study medication. Third, the infusion of distribution fluid was limited to a maximum volume of $1 \mathrm{~L}$. Finally, instead of removing the catheter immediately after administration of the study med- ication, the catheter remained in situ for a minimum of 24 hours after the administration of the study medication.

After the introduction of these four adjustment measures, no leakage was observed in 12 of the 14 remaining patients. Patient 13 showed a very minor leakage of $2.5 \%$ of the injected dose into the left-lower quadrant of the abdomen, and in patient 17, a moderate extraperitoneal leakage of $19 \%$ into the right flank and lower quadrant of the abdomen was seen.

The minimum time at which extraperitoneal leakage first was seen was as early as 4 hours af- 
ter the injection of the study medication, as defined on planar images. All 4 patients with extraperitoneal leakage had signs of leakage at 24 hours after the injection of the study medication. In none of these 4 patients were local side-effects caused by increased radiation outside the peritoneal cavity observed.

Radioactivity in blood serum was measured at time points 1, 6, 24, 48, 72, 96, and 168 hours postinjection of the study medication. As shown in Figure 2, the 3 patients with substantial leakage (patients 1, 3, and 17) had a 50\% lower peak activity in their blood at 48 hours after an i.p. injection. Furthermore, subsequent blood clearance of the radioactivity from 48 to 168 hours after the study drug administration was slower than in the group without leakage, indicating a slow release and reabsorption from the extraperitoneal compartment to the blood. There was a significant difference in the level of blood radioactivity (area under the curve) between the group of patients without leakage $(1.12 \pm 0.45)$ and the 3 patients with substantial leakage $(0.50 \pm 0.12 ; p<0.001)$.

\section{DISCUSSION}

Four (4) of the 17 patients demonstrated leakage after an i.p. administration of study medication. After adjusting the surgical procedure and the infusion method, leakage was reduced, but not completely prevented. Leakage of the study med- ication did not result in local toxicity resulting from radiation exposure.

To date, no studies or case reports about the leakage of i.p.-administered medication to extraperitoneal spaces, which was not subcutaneous (s.c.), have been described in the literature. Balink et al. ${ }^{14}$ reported a case of leakage into the s.c. tissue at the catheter site after i.p. therapy with ${ }^{32} \mathrm{P}$ during the treatment of malignant ascites. They detected the leakage because of regional side-effects after the administration of the study medication. As found in our study, the leakage of i.p. study medication does not always cause side-effects. This depends on the amount of leakage, the site of leakage, and the composition of the study medication. Different chemotherapeutic agents that have been used i.p. are classified as vesicant and irritant drugs, which may cause inflammation and/or tissue destruction. ${ }^{15}$

Major catheter problems, such as malfunction, did not occur in our study, but these have been reported in other studies. Several studies reported that $10 \%-50 \%$ of the patients treated with i.p. chemotherapy had catheter problems. Reported complications were malfunction of the catheter (inflow obstruction), infection, bowel perforation, ileus, abdominal pain, and an increased morbidity. ${ }^{16-19}$ Almost all these complications were reported in studies that used repeated administrations of the drugs. Because ${ }^{90} \mathrm{Y}$-muHMFG1 was given as a single dose, we used an adminis-

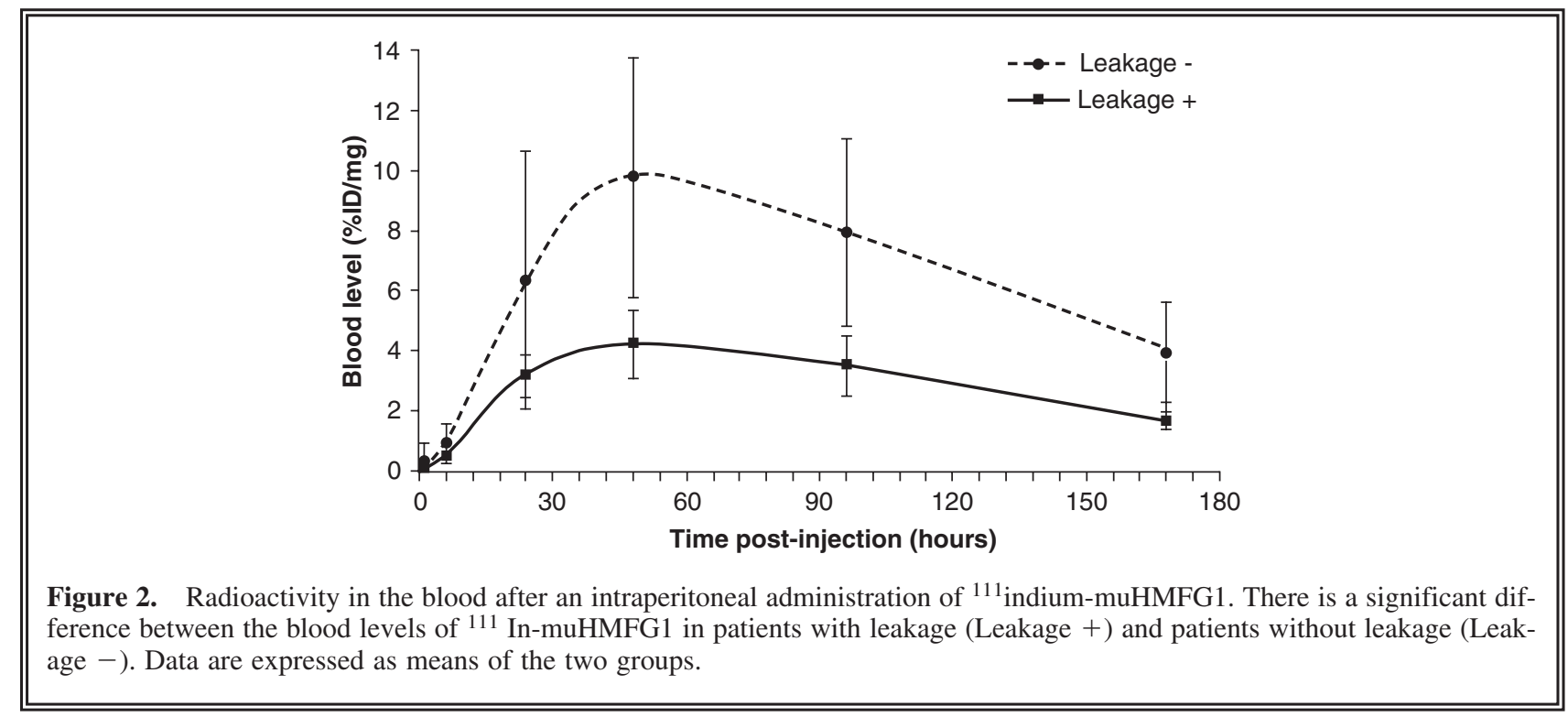


tration procedure, which was less invasive. Placement of the catheter during surgery and quick removal of the catheter after drug administration prevented extra surgical interventions and catheter complication. For repeated administration, the technique we have described in this paper is not appropriate, as it was designed for a one-time administration of i.p. therapy.

Recommendations for the use and placement of i.p. catheters for repeated drug administrations have been given by Markman and Walker ${ }^{20}$ and were the following: (i) the insertion of a fully implantable port attached to a single lumen venous silicone catheter on the inferior thorax at the midclavicular line; (ii) the catheter should be tunneled s.c., above the fascia, $6 \mathrm{~cm}$ lateral to the umbilicus, and pulled into the peritoneal cavity through a small hole the size of the catheter; and (iii) closure of the wound and peritoneal defect in several layers to prevent leakage. ${ }^{20}$

In our study, we restricted the use of distribution fluid in the i.p. cavity to $1 \mathrm{~L}$ at maximum after the first 3 patients. Several i.p. chemotherapy studies have used 1.5-2 liters of distribution fluid at maximum to facilitate the distribution of medication. ${ }^{5-7,21-25}$ The rationale behind minimizing the volume of distribution fluid was for a potentially lower i.p. pressure and, hence, to minimize the risk of leakage. Dedrick and Flessner ${ }^{26}$ demonstrated that an increase in irrigated area may be achieved in many patients by individualizing the distribution fluid volume and through the consideration of the patient's position. Obviouslyas in our study, both the surgical protocol and the distribution volume were modified-it is unclear which alteration had more impact on the reduction of leakage.

The main problem with regional therapy is the limited depth of penetration of the drugs directly into the tumor by free-surface diffusion. Experimental and clinical data support the conclusions that the high concentrations of drugs observed after delivery directly into the peritoneal cavity will only be relevant for patients with microscopic disease or very small-volume macroscopic cancer (e.g., largest residual mass less than 0.5-1.0 $\mathrm{cm}$ in diameter). ${ }^{27,28}$ In this context, the existence of leakage during i.p. therapy is of importance for the outcome of the treatment. Leakage may result in lower concentrations in the i.p. cavity and thereby potentially diminish the effects of the drug. If the surfaces of tumor nodules are not, or are inadequately exposed to the drug-containing solution, the rationale for regional administration is compromized and treatment failures could result. A recently published large international randomized trial of i.p. ${ }^{90} \mathrm{Y}$-muHMFG1 in ovarian cancer patients did not show a survival benefit between the active treatment and the standard treatment arm. ${ }^{10}$ This might, at least in part, be owing to leakage after i.p. therapy, possibly resulting in diminished therapeutic effectiveness.

Besides working as a regional therapy, i.p. therapy could also be effective in the systemic treatment of any extraperitoneal tumor localizations. Capillary uptake of the drug from the peritoneal cavity allows for the therapeutic concentrations to be achieved systematically, resulting in the control of extraperitoneal disease. As shown in Figure 2, the group with leakage shows a lower concentration in the blood, as compared to the group without leakage. The concentration in the peritoneal cavity is lower, which causes a lower gradient for the diffusion of the radioactivity and clearance out of the peritoneal cavity. Thus, when leakage occurs, it may affect not only the i.p. therapy, but also possible systemic effects.

Some phase III trials have documented the clinical benefit of i.p. cisplatin-based chemotherapy in the first-line treatment of optimally cytoreduced patients. ${ }^{5-7}$ The results of i.p. chemotherapeutic therapy when extraperitoneal leakage occurs are not yet known. The effect on efficacy and the local side-effects largely depends on the reabsorption from the extraperitoneal tissues.

\section{CONCLUSIONS}

In conclusion, significant extraperitoneal leakage of i.p.-administered medication may occur, which may result in decreased efficacy and potentially also in local side-effects. In view of the recently published clinical announcement of the National Cancer Institute to prefer a combination of i.p. and i.v. chemotherapy over i.v. chemotherapy alone in the first-line treatment of ovarian cancer ${ }^{29}$ and future developments of i.p. treatment of intra-abdominal cancer, the observations in this study need to be taken into consideration.

\section{ACKNOWLEDGMENTS}

This study was partly supported by Antisoma Research Ltd. (London, UK). 


\section{REFERENCES}

1. McGuire WP, Brady MF, Ozols RF. The Gynecologic Oncology Group experience in ovarian cancer. Ann Oncol 1999;10:29.

2. Weisberger A, Levine B, Storaasli JP. Use of nitrogen mustard in treatment of serous effusions of neoplastic origin. J Am Med Assoc 1955;159:1704.

3. Dedrick RL, Myers CE, Bungay PM, et al. Pharmacokinetic rationale for peritoneal drug administration in the treatment of ovarian cancer. Cancer Treat Rep 1978;62:1.

4. Markman M. Role of intraperitoneal chemotherapy in the front-line setting. J Clin Oncol 2003;21:145.

5. Alberts DS, Liu PY, Hannigan EV, et al. Intraperitoneal cisplatin plus intravenous cyclophosphamide versus intravenous cisplatin plus intravenous cyclophosphamide for stage III ovarian cancer. $N$ Engl J Med 1996;335:1950.

6. Armstrong DK, Bundy B, Wenzel L, et al. Intraperitoneal cisplatin and paclitaxel in ovarian cancer. $\underline{N \text { Engl }}$ J Med 2006;354:34.

7. Markman M, Bundy BN, Alberts DS, et al. Phase III trial of standard-dose intravenous cisplatin plus paclitaxel versus moderately high-dose carboplatin followed by intravenous paclitaxel and intraperitoneal cisplatin in small-volume stage III ovarian carcinoma: An intergroup study of the Gynecologic Oncology Group, Southwestern Oncology Group, and Eastern Cooperative Oncology Group. J Clin Oncol 2001;19:1001.

8. Du BA, Pfisterer J. Future options for first-line therapy of advanced ovarian cancer. Int J Gynecol Cancer 2005;15:42.

9. Markman M, Walker JL. Intraperitoneal chemotherapy of ovarian cancer: A review, with a focus on practical aspects of treatment. $J$ Clin Oncol 2006;24:988.

10. Verheijen RH, Massuger LF, Benigno BB, et al. Phase III trial of intraperitoneal therapy with yttrium-90-labeled HMFG1 murine monoclonal antibody in patients with epithelial ovarian cancer after a surgically defined complete remission. J Clin Oncol 2006;24:571.

11. Taylor-Papadimitriou J, Peterson JA, Arklie J, et al. Monoclonal antibodies to epithelium-specific components of the human milk fat globule membrane: Production and reaction with cells in culture. Int J Cancer 1981;28:17.

12. Dong Y, Walsh MD, Cummings MC, et al. Expression of MUC1 and MUC2 mucins in epithelial ovarian tumours. J Pathol 1997;183:311.

13. Taylor-Papadimitriou J, Burchell J, Miles DW, et al. MUC1 and cancer. Biochim Biophys Acta 1999;1455: 301.

14. Balink H, Sijmons EA, Zonnenberg BA, et al. Repetitive phosphorus-32 peritoneal instillations in a patient with malignant ascites. Clin Nucl Med 2003;28:545.

15. Ener RA, Meglathery SB, Styler M. Extravasation of systemic hemato-oncological therapies. Ann Oncol 2004;15:858.
16. Barakat RR, Sabbatini P, Bhaskaran D, et al. Intraperitoneal chemotherapy for ovarian carcinoma: Results of long-term follow-up. J Clin Oncol 2002;20:694.

17. Makhija S, Leitao M, Sabbatini P, et al. Complications associated with intraperitoneal chemotherapy catheters. Gynecol Oncol 2001;81:77.

18. Sakuragi N, Nakajima A, Nomura E, et al. Complications relating to intraperitoneal administration of cisplatin or carboplatin for ovarian carcinoma. Gynecol Oncol 2000;79:420.

19. Walker JL, Armstrong DK, Huang HQ, et al. Intraperitoneal catheter outcomes in a phase III trial of intravenous versus intraperitoneal chemotherapy in optimal stage III ovarian and primary peritoneal cancer: A Gynecologic Oncology Group Study. Gynecol Oncol 2006;100:27.

20. Markman M, Walker JL. Intraperitoneal chemotherapy of ovarian cancer: A review, with a focus on practical aspects of treatment. J Clin Oncol 2006;24:988.

21. Barakat RR, Almadrones L, Venkatraman ES, et al. A phase II trial of intraperitoneal cisplatin and etoposide as consolidation therapy in patients with stage II-IV epithelial ovarian cancer following negative surgical assessment. Gynecol Oncol 1998;69:17.

22. Guastalla JP, Lhomme C, Kerbrat P, et al. Phase II trial of intraperitoneal carboplatin in ovarian carcinoma patients with macroscopic residual disease at second-look laparotomy: A multicentre study of the French Federation Nationale des Centres de Lutte Contre le Cancer. Ann Oncol 1994;5:127.

23. Markman M, Brady MF, Spirtos NM, et al. Phase II trial of intraperitoneal paclitaxel in carcinoma of the ovary, tube, and peritoneum: A Gynecologic Oncology Group Study. J Clin Oncol 1998;16:2620.

24. Rothenberg ML, Liu PY, Braly PS, et al. Combined intraperitoneal and intravenous chemotherapy for women with optimally debulked ovarian cancer: Results from an intergroup phase II trial. $\underline{\text { Clin Oncol }}$ 2003;21:1313.

25. Topuz E, Eralp Y, Saglam S, et al. Efficacy of intraperitoneal cisplatin as consolidation therapy in patients with pathologic complete remission following front-line therapy for epithelial ovarian cancer: Consolidative intraperitoneal cisplatin in ovarian cancer. Gynecol Oncol 2004;92:147.

26. Dedrick RL, Flessner MF. Pharmacokinetic problems in peritoneal drug administration: Tissue penetration and surface exposure. J Natl Cancer Inst 1997;89:480.

27. Markman M. Intraperitoneal chemotherapy is appropriate first-line therapy for patients with optimally debulked ovarian cancer. Crit Rev Oncol Hematol 2001; 38:171.

28. Markman M. Intraperitoneal antineoplastic drug delivery: Rationale and results. Lancet Oncol 2003;4:277.

29. Clinical alert by the National Cancer Institute. Online document at: http://ctep.cancer.gov/highlights/ovarian. html Accessed Jan 5, 2006. 


\section{This article has been cited by:}

1. Pamela E Constantinou, Brian P Danysh, Neeraja Dharmaraj, Daniel D Carson. 2011. Transmembrane mucins as novel therapeutic targets. Expert Review of Endocrinology \& Metabolism 6:6, 835-848. [CrossRef]

2. S. J. Dawson, R. J. Hicks, V. Johnston, D. Allen, T. Jobling, M. Quinn, D. Rischin. 2011. Intraperitoneal distribution imaging in ovarian cancer patients. Internal Medicine Journal 41:2, 167-171. [CrossRef]

3. Richard E Beatson, Joyce Taylor-Papadimitriou, Joy M Burchell. 2010. MUC1 immunotherapy. Immunotherapy 2:3, 305-327. [CrossRef] 